Case Report

\title{
Cardiogenic Shock and Guillain-Barré Syndrome as the First Manifestations of Pheochromocytoma
}

\author{
Maryam Heidarpour, ${ }^{1}$ Nafiseh Sereshti, ${ }^{1}$ Davood Shafie $\mathbb{D}^{2}{ }^{2}$ Bijan Iraj, ${ }^{1}$ \\ and Hassan Rezvanian $(\mathbb{1})^{1}$ \\ ${ }^{1}$ Isfahan Endocrine and Metabolism Research Center, Isfahan University of Medical Sciences, Isfahan, Iran \\ ${ }^{2}$ Heart Failure Research Center, Isfahan Cardiovascular Research Institute, Isfahan University of Medical Sciences, Isfahan, Iran \\ Correspondence should be addressed to Davood Shafie; d.shafie87@gmail.com and Hassan Rezvanian; hasan.rezvanian@ \\ gmail.com
}

Received 9 November 2020; Revised 5 May 2021; Accepted 25 May 2021; Published 3 June 2021

Academic Editor: Osamu Isozaki

Copyright (c) 2021 Maryam Heidarpour et al. This is an open access article distributed under the Creative Commons Attribution License, which permits unrestricted use, distribution, and reproduction in any medium, provided the original work is properly cited.

\begin{abstract}
Febrile congestive heart failure is a rare first manifestation of pheochromocytoma. Herein, the case of a 31-year-old female with febrile congestive heart failure and subsequent cardiogenic shock is presented. After intensive care unit (ICU) admission and further evaluating the right adrenal mass observed in abdominal ultrasonography, the diagnosis of pheochromocytoma was confirmed. Then, she was scheduled for the right adrenalectomy. Before surgery, she complained of acute-onset progressive muscle weakness in the lower limbs, followed by the upper limbs. After further investigation, she was diagnosed with Guillain-Barré syndrome and treated with intravenous immunoglobulin (IVIG). She recovered well after the right adrenalectomy, and during the subsequent 18 months, the follow-up did not reveal any complications, and left ventricular function recovered to normal.
\end{abstract}

\section{Introduction}

Pheochromocytoma is a rare neuroendocrine tumor with the ability to synthesize and overproduce catecholamines, developed in the adrenal medulla $[1,2]$. Its clinical presentation is highly variable, but the classic triad is headache, sweating, and palpitation [3]. Less often, the tumor will cause severe cardiovascular complications such as myocardial infarction, arrhythmias, pericardial effusion, and congestive heart failure. Pheochromocytoma-induced cardiomyopathy is similar to Takotsubo cardiomyopathy and myocarditis $[4,5]$. Diagnosis of pheochromocytoma-related cardiomyopathies is often delayed because of the atypical presentation. Given the potential reversibility of the cardiomyopathy, early diagnosis and resection of the pheochromocytoma are critical, while delayed diagnosis may lead to irreversible cardiac remodeling and death [6].

On the other hand, Guillain-Barré syndrome (GBS) is an acute immune-mediated polyradiculoneuropathy. Symmetrical limb weakness and hyporeflexia are typical manifestations of the GBS. However, half of these patients present with cranial nerve involvement. There have been few case reports of cardiovascular involvement in patients with GBS [7-10]. Here, we describe a case of pheochromocytoma presented as febrile congestive heart failure with subsequent cardiogenic shock and GBS. Finally, we discuss our diagnostic and management insights and report the patient's condition over 18-month follow-up period.

\section{Case Presentation}

A 31-year-old female presented to the emergency room with severe dyspnea, palpitation, acute generalized abdominal pain, and vomiting without blood or bile in February 2019. Upon reviewing systems and past medical and surgical history, she admitted to a $5 \mathrm{~kg}$ weight loss over the past month. She had a cesarean section 18 months ago without any complications. She had no history of hypertension (HTN), diabetes mellitus (DM), and cigarette smoking. Her 
drug history was negative, especially for illegal drugs, such as methamphetamines and cocaine. Her symptoms started 56 hours before admission. Initial vital signs included a blood pressure of $80 / 50 \mathrm{mmHg}$, heart rate of 130 beats per minute $(\mathrm{bpm})$, respiratory rate of 30 per minute, $\mathrm{O}_{2}$ saturation of $81 \%$ on room air, and temperature of $38.1^{\circ} \mathrm{C}$. Physical examination revealed diffuse bilateral crackles but no decreased breath sounds at the base. Generally, the patient appeared confused and unstable. During the initial physical examination, she had a cardiac arrest, and cardiopulmonary resuscitation was done for 10 minutes, and it was successful. After that, she was sent to the intensive care unit (ICU). At this time, ECG showed sinus tachycardia, and transthoracic echocardiogram (TTE) demonstrated severe left ventricular (LV) systolic dysfunction with ejection fraction (EF) of $10 \%$, normal LV size, mild to moderate mitral regurgitation, mild tricuspid regurgitation, and moderate pericardial effusion. Laboratory data are shown in Table 1 . She received norepinephrine (starting infusion rates of $0.025 \mu \mathrm{g} / \mathrm{kg} / \mathrm{min}$ ), meropenem (500 mg IV every 8 hours), and ciprofloxacin (500 mg IV every 12 hours). On the fourth day, norepinephrine was discontinued, and hemodynamic stability was maintained even after discontinuation of vasopressor support. However, her fever continued to peak at $39^{\circ} \mathrm{C}$. Tracheal aspirate, blood, and urine cultures were taken, and abdominal ultrasonography was performed due to non-specific abdominal discomfort. Ultrasonography showed a welldefined hypoechoic mass $(44 \times 57 \mathrm{~mm})$ with internal vascularity in the right adrenal gland. In the next step, on the seventh day, abdominal computed tomography (CT) and catecholamine urine tests were requested for suspicion of an adrenal tumor. Abdominal CT showed a heterogeneous right adrenal mass measuring $47 \times 54 \mathrm{~mm}$ (Figure 1). The adrenal mass on the unenhanced images had a density of 36 Hounsfield units. Laboratory values were notable for a 24hour urine metanephrine of $2510 \mu \mathrm{g} /$ day (normal $<315 \mu \mathrm{g} /$ day), normetanephrine of $8657 \mu \mathrm{g} /$ day (normal $<670 \mu \mathrm{g}$ / day), adrenaline of $386 \mu \mathrm{g} /$ day (normal $<20 \mu \mathrm{g} /$ day), noradrenaline of $1044 \mu \mathrm{g} /$ day (normal $<90 \mu \mathrm{g} /$ day), and vanillylmandelic acid of $70 \mathrm{mg} /$ day (normal $<13.6 \mathrm{mg} /$ day). Indeed, all cultures were negative for infectious organisms. These findings were consistent with the diagnosis of pheochromocytoma. She was started on phenoxybenzamine $10 \mathrm{mg}$ twice a day, and the dosage was increased by $10 \mathrm{mg}$ every three days to a maximum dosage of $20 \mathrm{mg}$ twice a day. On the tenth day, she complained of acute-onset progressive muscle weakness in the lower limbs, followed by the upper limbs. The muscle strength examination showed severe weakness in four limbs with a Medical Research Council (MRC) scale of $1 / 5$ in proximal and $2 / 5$ in distal of the upper extremities and 2/5 in proximal and 2/5 in distal of the lower limbs. She had no spine sensory level. Deep tendon reflexes were absent. Upper motor neuron disorders and meningeal irritation signs were absent. Lumbar puncture for cerebrospinal fluid (CSF) analysis was performed, and study results are shown in Table 2 . Brain magnetic resonance imaging (MRI) was done and showed a normal finding except for a few foci of cortical and subcortical infarction. Electromyography (EMG) and nerve conduction velocity
TABLE 1: Laboratory test results.

\begin{tabular}{lccc}
\hline Test & Day 1 & Day 10 & Normal values \\
\hline FBS & 104 & 109 & Up to $100 \mathrm{mg} / \mathrm{dl}$ \\
BUN & 26 & 88 & $7-20 \mathrm{mg} / \mathrm{dl}$ \\
$\mathrm{Cr}$ & 1.3 & 1.6 & $0.8-1.3 \mathrm{mg} / \mathrm{dl}$ \\
$\mathrm{Na}$ & 138 & 152 & $135-145 \mathrm{mEq} / \mathrm{L}$ \\
$\mathrm{K}$ & 3.7 & 3.3 & $3.5-5 \mathrm{mEq} / \mathrm{L}$ \\
$\mathrm{Ca}$ & 8.4 & 7.8 & $8.5-10.5 \mathrm{mg} / \mathrm{dl}$ \\
$\mathrm{Ph}$ & 4.6 & 3.9 & $3.4-4.5 \mathrm{mg} / \mathrm{dl}$ \\
Mg & 2.2 & 1.7 & $1.7-2.2 \mathrm{mg} / \mathrm{dl}$ \\
Troponin & 17.9 & 54 & $\mathrm{Up} \mathrm{to} 0.4 \mathrm{ng} / \mathrm{ml}$ \\
D-Dimer & 5794 & - & $\mathrm{Up} \mathrm{to} 500 \mathrm{ng} / \mathrm{ml}$ \\
TSH & 1.4 & - & $0.35-0.5 \mathrm{mIU} / \mathrm{L}$ \\
WBC & 30.9 & 29.0 & $4.5-11 \times 10 \% / \mathrm{L}$ \\
Neut & $82.4 \%$ & $91 \%$ & $55-70 \%$ \\
Lymph & $7.6 \%$ & $4 \%$ & $20-40 \%$ \\
HB & 10.1 & 9.8 & $12-15 \mathrm{~g} / \mathrm{dl}$ \\
aPTT & 43 & 35 & $30-40 \mathrm{msec}$ \\
INR & 1.2 & 1.5 & Up to 1.1 \\
AST & 401 & 390 & $5-40 \mathrm{IU} / \mathrm{L}$ \\
ALT & 535 & 912 & $7-56 \mathrm{IU} / \mathrm{L}$ \\
ALP & 306 & 342 & $20-140 \mathrm{IU} / \mathrm{L}$ \\
ESR & 1 & 2 & $\mathrm{Up} \mathrm{to} 29 \mathrm{~mm} / \mathrm{hr}$ \\
CRP & 13 & 47 & Up to $10 \mathrm{mg} / \mathrm{L}$ \\
Alb & 3.3 & 3.2 & $3.3-5.4 \mathrm{~g} / \mathrm{dl}$ \\
\hline BUN & & &
\end{tabular}

BUN: blood urea nitrogen; Cr: creatinine; Na: sodium; K: potassium; Ca: calcium; $\mathrm{Ph}$ : phosphorus; $\mathrm{Mg}$ : magnesium; TSH: thyroid-stimulating hormone; WBC: white blood cell; Neut: neutrophil; Lymph; lymphocyte; HB: hemoglobin; aPTT: activated partial thromboplastin time; INR: international normalized ratio; AST: aspartate aminotransferase; ALT: alanine aminotransferase; ALP: alkaline phosphatase; ESR: erythrocyte sedimentation rate; CRP: C-reactive protein; Alb: albumin.

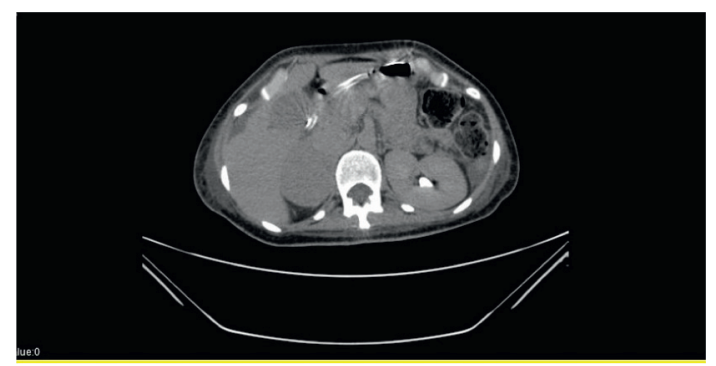

FIgure 1: Abdominal CT scan of the patient shows heterogeneous right adrenal mass.

(NCV) showed acute predominantly motor axonal polyneuropathy. Therefore, the diagnosis of GBS was made, and the patient was treated with intravenous immunoglobulin (IVIG) $2 \mathrm{~g} / \mathrm{kg}$ divided over five days. After that, muscle weakness gradually improved. On the fourteenth day, propranolol was prescribed with a dose of $10 \mathrm{mg}$ every 8 hours. Then, the right adrenalectomy was performed without any surgical complications, and the postoperative histopathologic report was in agreement with a diagnosis of pheochromocytoma. No complications were reported in the postoperative period. Predischarge TTE showed LVEF of $40 \%$, normal LV size, mild mitral regurgitation, mild tricuspid regurgitation, and no pericardial effusion. She was followed in an outpatient setting regularly. Two weeks after adrenalectomy, the patient had no evidence of the disease. 
TABLE 2: CSF analysis results.

\begin{tabular}{lcc}
\hline Test & Patient's values & Normal values \\
\hline CSF glucose & 68 & $>50 \%$ serum \\
Serum glucose & 118 & Up to 200 mg/dl(random sampling) \\
Protein & 0.48 & $0.15-0.45 \mathrm{~g} / \mathrm{L}$ \\
WBC & 0 & $<5\left(\mathrm{~mm}^{3}\right)$ \\
RBC & 3 & $0\left(\mathrm{~mm}^{3}\right)$ \\
Viral PCR (HSV 1 and 2, VZV, and Enterovirus) & Negative & Negative \\
\hline
\end{tabular}

CSF: cerebrospinal fluid; WBC: white blood cell; RBC: red blood cell; HSV: herpes simplex virus; VZV: varicella-zoster virus.

Laboratory values were notable for a 24-hour urine metanephrine of $107 \mu \mathrm{g} /$ day (normal $<315 \mu \mathrm{g} /$ day), normetanephrine of $210 \mu \mathrm{g} /$ day (normal $<670 \mu \mathrm{g} /$ day), adrenaline of $15 \mu \mathrm{g} /$ day (normal $<20 \mu \mathrm{g} /$ day), noradrenaline of $34 \mu \mathrm{g} /$ day (normal $<90 \mu \mathrm{g} /$ day), and vanillylmandelic acid of $7.1 \mathrm{mg} /$ day (normal $<13.6 \mathrm{mg} /$ day). Today, 18 months after hospital discharge, her general condition is good, and the catecholamine urine test results are negative for pheochromocytoma. The latest echocardiography demonstrates the LVEF to be $50 \%$, and other echocardiographic measurements are within the normal values.

\section{Discussion}

This case report presented a young female with complicated pheochromocytoma having unusual presentations of hypotension, cardiac arrest, cardiomyopathy, and fever. Also, she was complicated with GBS in the course of hospitalization.

Pheochromocytoma exhibits variable characteristics with diverse presentations. Although it is rare, it is a fatal emergency, and diagnosis can be extremely challenging. Since our case presented predominantly with cardiac problems, it is mandatory to discuss cardiovascular complications of pheochromocytoma in detail. Pheochromocytoma usually manifests as severe hypertension, circulatory shock, arrhythmias, myocardial injury, and cardiomyopathy - the excessive catecholamine exposure of myocardium results in toxic effects like myocardial necrosis and cardiomyopathy [11]. The prevalence of cardiomyopathy in pheochromocytoma has been reported to be about $11 \%$ [12]. Of course, we must also consider cases of pheochromocytoma that have not been diagnosed and reported. In these patients, cardiomyopathy may be presented as a global or focal abnormality. Interestingly, a complete reversal of pheochromocytoma cardiomyopathy has been reported in a short period of as much as eight days, following the tumor's removal [13].

Approximately $50 \%$ of patients with pheochromocytoma have a hypertensive crisis; however, $5-15 \%$ are normotensive, and in rare instances, these patients have hypotension. Bergland et al. reported in a review of 539 cases of pheochromocytoma that $2 \%$ of the cases presented with hypotension and shock [14]. Possible hypotension mechanisms in pheochromocytoma are hypovolemia, desensitization of blood vessels to catecholamine, reduced vascular resistance due to high epinephrine release from the tumor, myocardial contractile dysfunction, and hemorrhagic necrosis of the tumor [14].
Fever is also an uncommon presentation in these patients. In a case series of 25 patients, $12 \%$ presented with fever [15]. The excessive release of epinephrine has been postulated as a cause of fever by combining hypermetabolism and cutaneous vasoconstriction [16]. Of course, infectious causes and inflammatory disease should always be considered in all patients with febrile pericarditis and it should be evaluated first.

The further exciting twist in our case is the co-occurrence of GBS and pheochromocytoma. Although this is not impossible, it rarely happens. The average annual incidence of GBS was 1 per 100000 patient-years, while the incidence of pheochromocytoma are approximately 0.8 per 100000 patient-years $[5,17]$. Abdel-Salam et al. reported the case of GBS with subsequent pheochromocytoma [17].

Indeed, Ahmad et al. reported that urine and plasma catecholamine levels were elevated in patients with GBS who had autonomic instability [18]. However, in our case, the clinical manifestations of GBS occurred after the diagnosis of pheochromocytoma, and cardiac complications began to improve after adrenalectomy. Although the exact cause of GBS remains to be clearly understood, previous studies indicated an association between immunological stressors (such as viral infection and vaccination) and GBS. Our case denied any flu-like symptoms or vaccination in the preceding two weeks. Therefore, the question arises that can hypersecretion of catecholamine and pro-inflammatory states in pheochromocytoma provoke GBS?

In our case, considering the young age, female sex, fever and dyspnea as first manifestations, normal ECG except for sinus tachycardia, lack of traditional cardiovascular risk factors, and negative drug history for cocaine, coronary heart disease was less suspected.

Fortunately, the patient was diagnosed and treated at a referral center despite having a large tumor and catastrophic symptoms and signs. Finally, we emphasize that the clinical manifestations of pheochromocytoma depend on the various hormone secretion, and the diversity of this matter should be considered in any clinical scenario. However, whether there is a direct link between GBS and pheochromocytoma is a mystery that needs to be answered with more detailed studies.

\section{Data Availability}

No data were used to support this study.

\section{Conflicts of Interest}

The authors declare that they have no conflicts of interest. 


\section{References}

[1] J. W. Lenders, G. Eisenhofer, M. Mannelli, and K. Pacak, "Phaeochromocytoma," The Lancet, vol. 366, no. 9486, pp. 665-675, 2005.

[2] R. Hodin, C. Lubitz, R. Phitayakorn, and A. Stephen, "Diagnosis and management of pheochromocytoma," Current Problems in Surgery, vol. 51, no. 4, pp. 151-187, 2014.

[3] J. T. Adler, G. Y. Meyer-Rochow, H. Chen et al., "Pheochromocytoma: current approaches and future directions," The Oncologist, vol. 13, no. 7, pp. 779-793, 2008.

[4] P. A. Marcovitz, P. Czako, S. Rosenblatt, and S. S. Billecke, "Pheochromocytoma presenting with Takotsubo syndrome," Journal of Interventional Cardiology, vol. 23, no. 5, pp. 437442, 2010.

[5] M. Heidarpour, M. A. Haghighatpanah, H. Rezvanian et al., "Polyserositis: an extremely rare life-threatening manifestation of pheochromocytoma," Case Reports in Endocrinology, vol. 2020, Article ID 8814699, 3 pages, 2020.

[6] R. Zhang, D. Gupta, and S. G. Albert, "Pheochromocytoma as a reversible cause of cardiomyopathy: analysis and review of the literature," International Journal of Cardiology, vol. 249, pp. 319-323, 2017.

[7] J. S. Finkelstein and B. H. Melek, "Guillain-barré syndrome as a cause of reversible cardiomyopathy," Texas Heart Institute Journal, vol. 33, pp. 57-59, 2006.

[8] K. Iga, Y. Himura, C. Izumi et al., "Reversible left ventricular dysfunction associated with guillain-barre syndrome. An expression of catecholamine cardiotoxicity?" Japanese Circulation Journal, vol. 59, no. 4, pp. 236-240, 1995.

[9] A. Hiraga, K. Nagumo, K. Suzuki, Y. Sakakibara, and S. Kojima, "A patient with Guillain-Barre syndrome and recurrent episodes of ST elevation and left ventricular hypokinesis in the anterior wall [in Japanese]," No To Shinkei, vol. 55, pp. 517-520, 2003.

[10] M. Boon, P. J. Dennesen, and R. F. Veldkamp, "A rare stress cardiomyopathy in a patient with Guillain-Barré syndrome," The Netherlands Journal of Medicine, vol. 74, no. 2, pp. 86-88, 2016.

[11] P. Sethi and C. D. Peiris, "A review of catecholamine associated cardiomyopathies and channelopathies," Cureus, vol. 12, no. 2, p. e6957, 2020.

[12] A. Giavarini, A. Chedid, G. Bobrie, P.-F. Plouin, A. Hagège, and L. Amar, "Acute catecholamine cardiomyopathy in patients with phaeochromocytoma or functional paraganglioma," Heart, vol. 99, no. 19, pp. 1438-1444, 2013.

[13] M. Salathe, P. Weiss, and R. Ritz, "Rapid reversal of heart failure in a patient with phaeochromocytoma and catecholamine-induced cardiomyopathy who was treated with captopril," Heart, vol. 68, no. 11, pp. 527-528, 1992.

[14] B. E. Bergland, "Pheochromocytoma presenting as shock," The American Journal of Emergency Medicine, vol. 7, no. 1, pp. 44-48, 1989.

[15] W.-B. Liao, C.-F. Liu, C.-W. Chiang, C.-T. Kung, and C.-W. Lee, "Cardiovascular manifestations of pheochromocytoma," The American Journal of Emergency Medicine, vol. 18 , no. 5, pp. $622-625,2000$.

[16] S. Fukumoto, T. Matsumoto, S.-I. Harada, J. Fujisaki, M. Kawano, and E. Ogata, "Pheochromocytoma with pyrexia and marked inflammatory signs: a paraneoplastic syndrome with possible relation to interleukin-6 production*," The Journal of Clinical Endocrinology \& Metabolism, vol. 73, no. 4, pp. 877-881, 1991.
[17] F. Abdel-Salam, A. P. Basu, S. Johnson, and A. M. Devlin, "Suspected pheochromocytoma in a patient with guillainbarré syndrome," Pediatrics, vol. 191, no. 3, pp. 955-958, 2013.

[18] J. Ahmad, A. Kham, and M. Siddiqui, "Estimation of plasma and urinary catecholamines in Guillain-Barre syndrome," Japanese Journal of Medicine, vol. 24, no. 1, pp. 24-29, 1985. 